\title{
Predictive Failure of Cylindrical Coatings Using Weibull Analysis
}

\author{
Brian L. Vlcek \\ Georgia Southern University, Statesboro, Georgia, USA \\ Robert C. Hendricks and Erwin V. Zaretsky \\ National Aeronautics and Space Administration, Glenn Research Center, Cleveland, Ohio, USA
}

\begin{abstract}
Rotating, coated wiping rollers used in a high-speed printing application failed primarily from fatigue. Two coating materials were evaluated: a hard, cross-linked, plasticized polyvinyl chloride (PVC) and a softer, plasticized PVC. A total of 447 tests was conducted with these coatings in a production facility. The data were evaluated using Weibull analysis. The softer coating produced more than twice the life of the harder cross-linked coating and reduced the wiper replacement rate by two-thirds, resulting in minimum production interruption.
\end{abstract}

Keywords Coatings, Fatigue, Printing, Weibull

Intaglio printing is used in high-quality applications where a desired texture is imparted to the finished print product along with the ink. In this printing process, ink is applied to the entire surface of the printing plate, although ink is ultimately desired only in the engraved valleys of the plate. The excess ink on the flat plateaus between the engraved valleys (known in the trade as the "lands") must be removed before it is transferred to the printed product. The paper is then squeezed against the plate with such force that ink is transferred from the capillary-like engraved lines, and the surface of the paper is textured and/or deformed.

A coated cylinder is used as a wiping roller to remove the excess ink from the printing plates. The wiper rotates and oscillates from side to side as excess ink is removed from the printing plate surface before impressions are made. On press, the wiping roller sits in a tank of caustic solution where ink is removed physically, by brushes and blades located in the tank, and chemically, from the wiper surface.

Received 24 September 2002; accepted 24 September 2002. Address correspondence to Robert C. Hendricks, MS 5/9, NASA Glenn Research Center, 21000 Brookpark Road, Cleveland, OH 44135. E-mail: robert.c.hendricks@grc.nasa.gov
In an application of high-speed Intaglio printing, the surface coating of rotating cylindrical wiping rollers fails because of fatigue and/or misuse. Microscopic and visual inspections of failed wiper surfaces show that the primary mode of failure is fatigue. Fields of cracks 10 to $30 \mu \mathrm{m}$ wide and 100 to $300 \mu \mathrm{m}$ long form on the surface of failed coatings. Figure 1 is a micrograph of a failed wiper coating surface shown at a magnification of 100 . Excess ink that has become entrapped in the fatigue cracks is transferred to the printing plates, imprinting the fatigue crack images on the finished product. The failure criterion is artistically driven and is based upon the appearance of the printed product. Thus, wipers are removed not at the first appearance of fatigue cracks on the surface but at the first visual appearance of a crack image transferred to the printed product.

Based upon the fatigue criterion, a test was conducted to determine the endurance of the roller coatings. The standard coating used was manufactured in-house by the user and was a hard, cross-linked, plasticized polyvinyl chloride (PVC) thermoset. The candidate replacement coating was a softer plasticized PVC. The objectives of the research were to (a) determine the fatigue life of each of the coatings, (b) determine the rate of coating replacement due to failure, and (c) minimize the impact of early failures.

\section{STATISTICAL METHOD}

\section{Weibull Method}

Waloddi Weibull (1939a, 1939b, 1951) developed a method for statistically evaluating the fracture strength of materials based upon small population sizes. He stated that the dispersion in material strength for a group of homogeneous test specimens can be expressed by

$$
\ln \ln \frac{1}{S}=e \ln \left[\frac{X}{X_{\beta}}\right]
$$

where $S$ is the probability of survival as a fraction $(0<S<1), X$ is the stress or strength $\left(\mathrm{N} / \mathrm{m}^{2}\right.$ or psi), and $X_{\beta}$ is the characteristic 


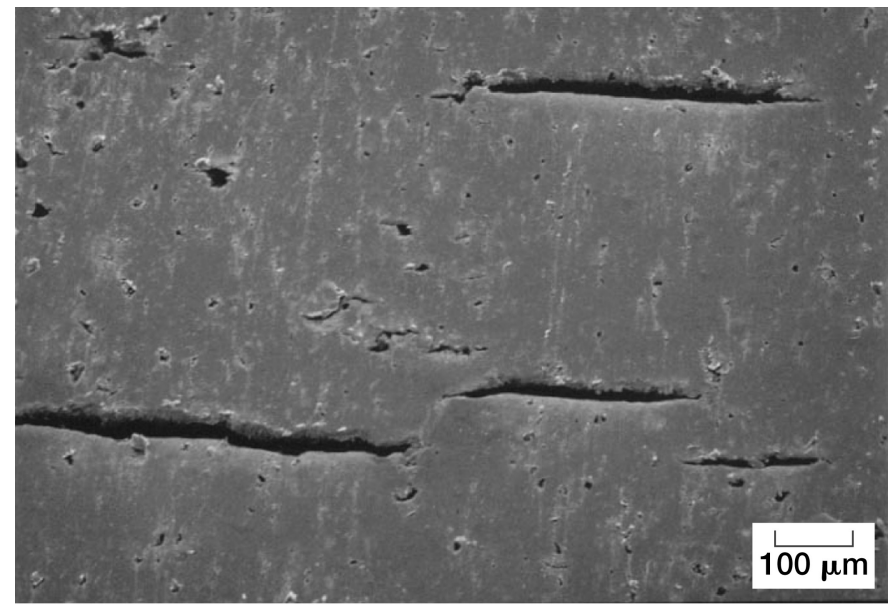

FIGURE 1

Fatigue cracks on surface of failed wiper roller coating.

stress or strength $\left(\mathrm{N} / \mathrm{m}^{2}\right.$ or psi). By plotting the ordinate scale as $\ln \ln (1 / S)$ and the abscissa scale as $\ln X$, a cumulative distribution will plot as a straight line, which is called a "Weibull plot." Usually, the ordinate is graduated in statistical percent of specimens failed. Figure 2 is a generic Weibull plot with some of the values of interest indicated.

The Weibull plot can be used to evaluate phenomena that result in a statistical distribution. The tangent of the resulting plot, called the Weibull slope and designated by $e$ or $m$, defines the statistical distribution. Weibull slopes of 1,2, and 3.57 represent exponential, Raleigh, and Gaussian (normal) distributions, respectively. Lundberg and Palmgren (1947) were the first to use Weibull analysis to predict the lives of rolling-element bearings.

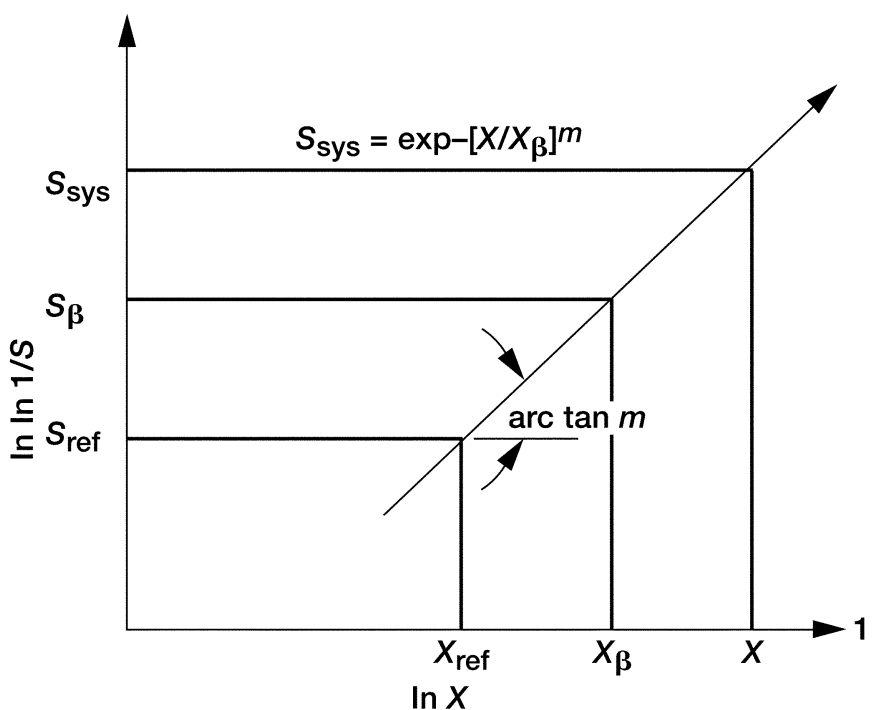

FIGURE 2

Sketch of Weibull plot where (Weibull) slope or tangent of line is $m, \mathbf{S}_{\beta}$ is probability of survival of 36.8 percent at which $X=X_{\beta}$ or $X / X_{\beta}=1$ (from Melis et al., 1999).
The statistical method for plotting and evaluating data using Weibull analysis was developed by Johnson (1951, 1964). It is assumed that a straight line is representative of the data, even though in many cases the data loci are not linear. Using the method of least squares, a straight line is drawn through an array of points on each plot. From this straight line, two significant lives are obtained for comparison: these are the lives at which 10 and 50 percent of the specimens have failed and are designated $L_{10}$ and $L_{50}$, respectively. The failure index given with each plot indicates the number of failed specimens of those tested. The confidence number is a comparison of the repeatability of the test results. It is dependent upon the number of failures in each group of specimens, the ratio of the lives being compared, and the Weibull slope of each group. A confidence number of 90 percent indicates that 90 out of 100 times the qualitative results would be the same if the tests were to be repeated.

From the Weibull plot, the values of $e$ and $X_{\beta}$ are determined. With these values known, it is possible to determine the failure rate for any time increment from Equation (1).

\section{Premature Failures}

Wiper coatings were known to fail prematurely in production as a result of accidents such as blade cuts rather than from fatigue. In an attempt to isolate the fatigue characteristics of both wiper materials being evaluated, early failures were intentionally converted to suspensions (removed from press for reasons other than for a fatigue-failed surface). A life was selected at the point below which all failures were converted to suspensions. This life was determined after finding a natural break in the data set and/or after observing distinct differing regions in the Weibull plots. The probability of failure was determined for this new data set and plotted on Weibull paper as a function of life. Weibull slope and the $L_{10}$ and the $L_{50}$ lives were again determined from the plot.

\section{SPECIMEN AND PROCEDURE}

\section{Materials}

Two wiper coatings were evaluated in production. The $\mathrm{P}$-series coating was a hard, cross-linked, plasticized PVC thermoset. High-temperature curing and thermal-driven crosslinking resulted in a Shore D 48 hardness. For the A-series coating, high-temperature curing resulted in a plasticized PVC surface with a Shore D 28 hardness. Both coating materials were produced in-house by the user and were utilized on the press to produce the backside of a printed surface.

\section{Test Method}

The test method was driven by production printing practices. The two wiper coatings ( $\mathrm{P}$ and $\mathrm{A}$ ) were evaluated on six backpresses as part of normal production usage (a backpress is a press that prints on the backside of the product). The wiper coatings were inspected upon removal of the wipers from the presses. 
To refurbish the cylindrical wipers, the coatings were removed and/or stripped, and the wipers were recoated for future use. The wiper cores that could not be refurbished were repaired or discarded.

Three hundred and forty-three A-series coated wipers were evaluated over a 9-month period. Of the 343 wiper surfaces, 265 were reported as failures and 78 as suspensions. Of the 104 P-series coated wipers tested on the same 6 backpresses during the same time period, 85 failed and 19 were suspended.

The analysis of the data involves a single backpress and a group of six backpresses and is discussed in the following section. The impact of premature failures is also investigated by intentionally suspending failures below a certain threshold and determining the effect of material and failure distribution on replacement rate.

\section{RESULTS AND DISCUSSION}

Rotating, cylindrical, coated wiping rollers used in Intaglio printing failed primarily because of fatigue or misuse. Two coating materials were evaluated in a production facility that had six printing presses dedicated to printing the backside of a product. The standard coating used was a hard, cross-linked, plasticized PVC thermoset designated as P-series. The candidate replacement coating was a plasticized PVC designated as A-series. A total of $104 \mathrm{P}$-series coatings and 343 A-series coatings was tested over 9 months and were evaluated during printing production.

A Weibull analysis was performed on the results to determine which of the wiper coatings was significantly better. The Weibull slope $L_{10}$ and $L_{50}$ lives were determined for each material. Confidence levels comparing the two materials on all six backpresses were determined.

\section{Data from a Single Backpress}

These data are from only one backpress and the two materials (A and P). Of the $24 \mathrm{P}$-series coatings, 21 were failures and 3

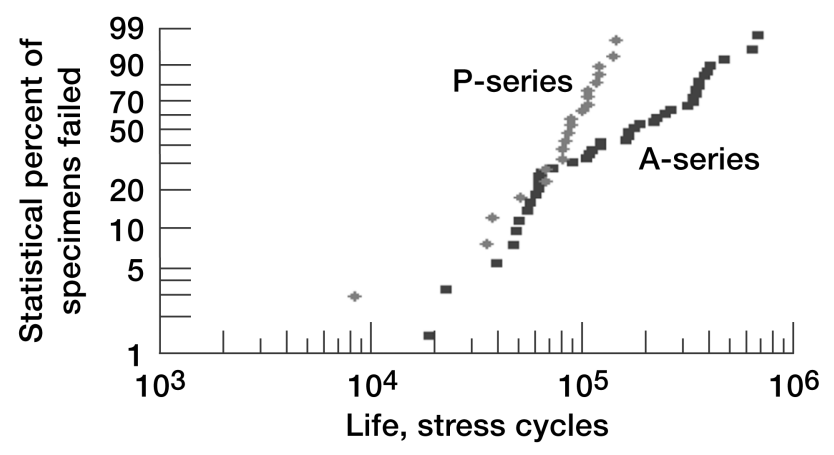

FIGURE 3

Weibull plot of A-series and P-series wiper coating data from single backpress. Failure index: A-series, 42/56; P-series, $21 / 24$.
TABLE 1

Summary of Weibull Results for P-Series and A-Series Coating Data from Single Backpress

\begin{tabular}{|c|c|c|}
\hline & \multicolumn{2}{|c|}{$\begin{array}{l}\text { Wiper coating material } \\
\text { on single backpress }\end{array}$} \\
\hline & P-series & A-series \\
\hline \multicolumn{3}{|l|}{ Life ${ }^{a}$ stress cycles } \\
\hline$L_{10}$ & 30169 & 46595 \\
\hline$L_{50}$ & 86902 & 199145 \\
\hline Average life,$^{b}$ stress cycles & 81460 & 194294 \\
\hline Weibull slope, $e$ & 1.78 & 1.30 \\
\hline Failure index ${ }^{c}$ & $21 / 24$ & $42 / 56$ \\
\hline Confidence number, ${ }^{d}$ percent & 77 & 77 \\
\hline
\end{tabular}

${ }^{a}$ Lives at which 10 and 50 percent of specimens failed.

${ }^{b}$ Total life of all specimens (including suspensions) divided by number of specimens (including suspensions).

${ }^{c}$ Number (numerator) of failures out of total tested (denominator).

${ }^{d}$ Percent of time qualitative test results will be same if test is repeated.

were suspensions. Of the 56 A-series coatings, 42 were tested to failure and 14 were suspended. The results are shown in Figure 3 and are summarized in Table 1. Of the two materials, the A-series coating is the longer lived by a factor of 1.5 at the $L_{10}$ life. The method of Johnson (1964) was used to obtain a confidence level of 77 percent.

\section{Data from Six Backpresses}

The data from the six backpresses were combined for each of the two coatings (P-series and A-Series) tested. Of the 104 P-series coated wipers tested, 85 failed and 19 were suspended. Of the 343 A-series coatings, 265 failed and 78 were suspended. The results of these tests are shown in Figure 4 and are summarized in Table 2. The $L_{10}$ life of the A-series coating was approximately 2.3 times that of the P-series. The confidence number for these data was 99 percent.

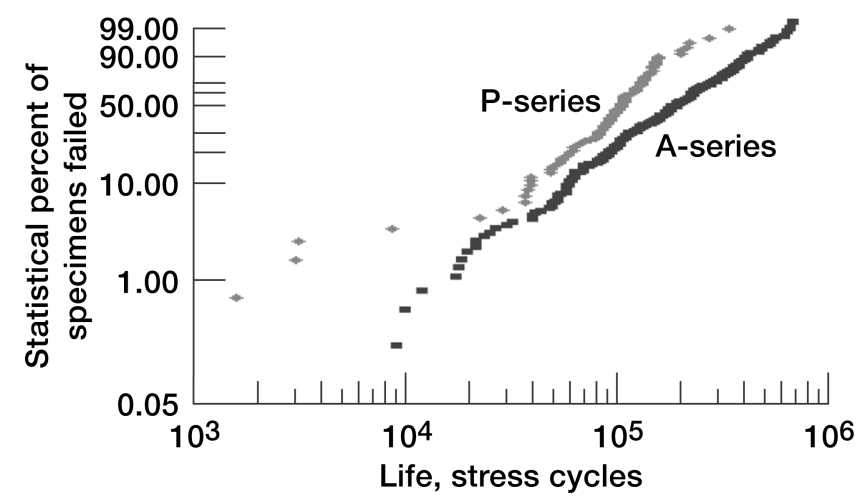

FIGURE 4

Weibull plot of A-series and P-series wiper coating data combined from six backpresses. Failure index: A-series, 265/343; P-series, 85/104. 
TABLE 2

Summary of Weibull Results for P-Series and A-Series Coating Data Combined from Six Backpresses

\begin{tabular}{|c|c|c|}
\hline & \multicolumn{2}{|c|}{$\begin{array}{l}\text { Wiper coating material } \\
\text { on all backpresses }\end{array}$} \\
\hline & P-series & A-serie \\
\hline \multicolumn{3}{|l|}{ Life, ${ }^{a}$ stress cycles } \\
\hline$L_{10}$ & 25402 & 58774 \\
\hline$L_{50}$ & 102886 & 194179 \\
\hline Average life, ${ }^{b}$ stress cycles & 95168 & 186206 \\
\hline Weibull slope, $e$ & 1.35 & 1.58 \\
\hline Failure index ${ }^{c}$ & $85 / 104$ & $265 / 34$ \\
\hline Confidence number, ${ }^{d}$ percent & 99 & \\
\hline
\end{tabular}

${ }^{a}$ Lives at which 10 and 50 percent of specimens failed.

${ }^{b}$ Total life of all specimens (including suspensions) divided by number of specimens (including suspensions).

${ }^{c}$ Number (numerator) of failures out of total tested (denominator).

${ }^{d}$ Percent of time qualitative test results will be same if test is repeated.

\section{Backpress Data Evaluation with Enforced Suspensions}

As was experienced in production, early failures were exhibited in these data. To evaluate the effect of these early failures on the life of each coating population, failures below a clear, natural break in the data set were suspended. These early failures were assumed to result from mishandling by the operator and not from fatigue failure. For the P-series data on all backpresses, all failures below 50,000 impressions were manually converted to suspensions. The six longest lives were also suspended, resulting in a comparatively straight line (Figure 5). The A-series data set was suspended for values less than 90,000 impressions. The Weibull slope and reliability for these suspended results are provided in Table 3. The ratio of the $L_{10}$ lives was 1.6. The confidence number was 96 percent. Eliminating the early failures from the analysis did not have a significant effect on the relative

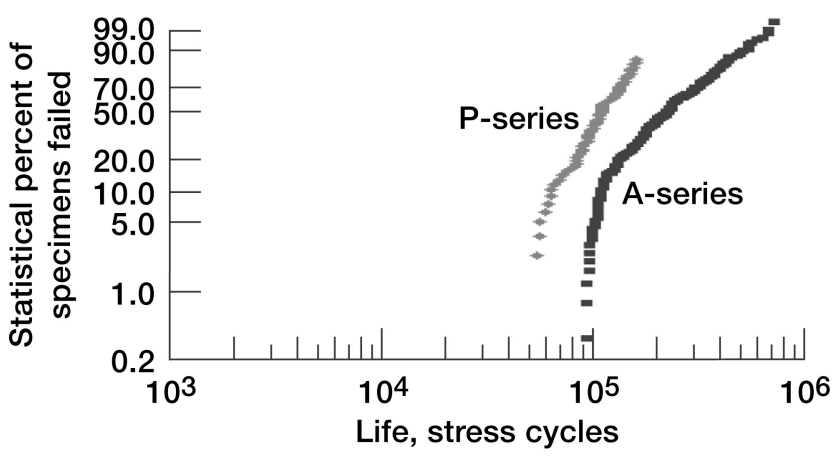

FIGURE 5

Weibull plot of A-series and P-series wiper coating data combined from six backpresses and suspensions. Failure index: A-series, 204/343; P-series, 64/104.
TABLE 3

Summary of Weibull Results for P-Series and A-Series Coating Combined Data

[Includes enforced suspensions from six backpresses.]

\begin{tabular}{|c|c|c|}
\hline & \multicolumn{2}{|c|}{$\begin{array}{l}\text { Wiper coating material } \\
\text { on all backpresses }\end{array}$} \\
\hline & P-series & A-series \\
\hline \multicolumn{3}{|l|}{ Life ${ }^{a}$ stress cycles } \\
\hline$L_{10}$ & 69360 & 112757 \\
\hline$L_{50}$ & 113390 & 247548 \\
\hline Average life, stress cycles ${ }^{b}$ & 95168 & 186206 \\
\hline Weibull slope, $e$ & 3.83 & 2.40 \\
\hline Failure index ${ }^{c}$ & $64 / 104$ & $204 / 343$ \\
\hline Confidence number, ${ }^{d}$ percent & 96 & 96 \\
\hline
\end{tabular}

${ }^{a}$ Lives at which 10 and 50 percent of specimens failed.

${ }^{b}$ Total life of all specimens (including suspensions) divided by number of specimens (including suspensions).

${ }^{c}$ Number (numerator) of failures out of total tested (denominator).

${ }^{d}$ Percent of time qualitative test results will be same if test is repeated.

life of the coatings, which suggested that early failures were coating related.

\section{Wiper Replacement Protocol}

Herein, assume that individual presses make 8000 impressions an hour. Thus, an individual coating receives 8000 stress cycles per hour or 64,000 stress cycles in an 8 -hr shift. Using Equation (1) and the data from Figure 4 and Table 2 for the P-series coating, 31 percent of the coatings would fail before the 8-hr shift were completed. If the remaining wipers were not replaced before the next 8 -hr shift, an additional 30 percent of the original coatings would fail and need to be replaced.

For the A-series coating data from Figure 4 and Table 2, the replacement rate for an 8-hr shift was 11 percent or one-third that of the P-series coating. Over two shifts, or $16 \mathrm{hr}, 30$ percent of the total coatings would be replaced. The replacement rate on the second 8-hr shift would be twice that of the first 8-hr shift. It can be reasonably concluded that by replacing the P-series coating with the A-series coating and replacing all wipers after each 8-hr shift, the failure rate of the wipers during an 8-hr shift could be reduced by approximately two-thirds. A cost-benefit analysis would be required before making a business decision based on these findings.

\section{SUMMARY OF RESULTS}

Rotating, cylindrical, coated wiping rollers used in Intaglio printing failed primarily by fatigue. Two coating materials were evaluated in a production facility that had six printing presses dedicated to printing the backside of a product. In a 9-month period, over 400 wiper coatings were evaluated in printing production. The P-series coating was a hard, cross-linked, 
plasticized polyvinyl chloride (PVC) thermoset. The candidate replacement coating was a softer, plasticized PVC designated as A-series. A total of 104 P-series coatings and 343 A-series coatings was tested. The following results were obtained:

1. The A-series coating had over twice the life of the P-series coating and a confidence level of 99 percent. During an 8-hr shift, the wiper replacement rate was reduced by approximately two-thirds.

2. A minimum failure rate of 11 percent was attained by replacing all A-series coated wipers after each 8-hr shift, resulting in minimum production interruption.

3. The suspension of early failures in the analysis did not significantly alter the ratio of $L_{10}$ lives (life at which 10 percent of the specimens have failed). This result suggested that early failures were coating and not operator related.

\section{REFERENCES}

Johnson, L. G. 1951. The median ranks of sample values in their population with an application to certain fatigue studies. Industrial Mathematics 2:1-9.

Johnson, L. G. 1964. The Statistical Treatment of Fatigue Experiments. Elsevier Publishing Co., Amsterdam.

Lundberg, G., and Palmgren, A. 1947. Dynamic Capacity of Rolling Bearings. Acta Polytechnica, Mechanical Engineering Series, vol. 1, no. 3, Stockholm.

Melis, M. E., Zaretsky, E. V., and August, R. 1999. Probabilistic analysis of aircraft gas turbine disk life and reliability. AIAA Trans., J. Propulsion and Power 15:658-666.

Weibull, W. 1939a. A Statistical Theory of the Strength of Materials. Ingeniors Vetanskaps Akademien-Handlinger, no. 151.

Weibull, W. 1939b. The Phenomenon of Rupture in Solids. Ingeniors Vetanskaps Akademien-Handlinger, no. 153.

Weibull, W. 1951. A statistical distribution function of wide applicability. Trans. ASME, J. Applied Mechanics 18:293-297. 

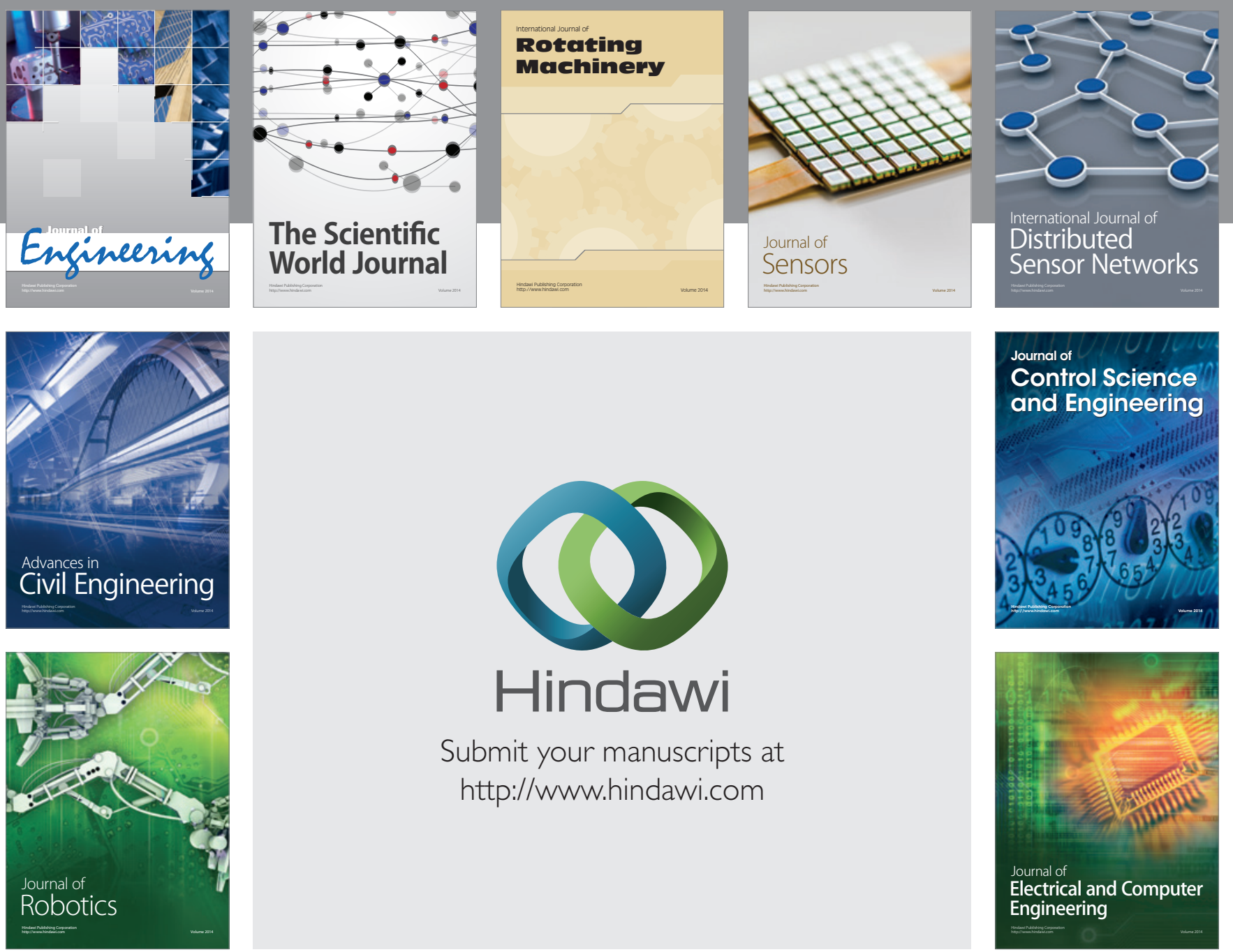

Submit your manuscripts at

http://www.hindawi.com
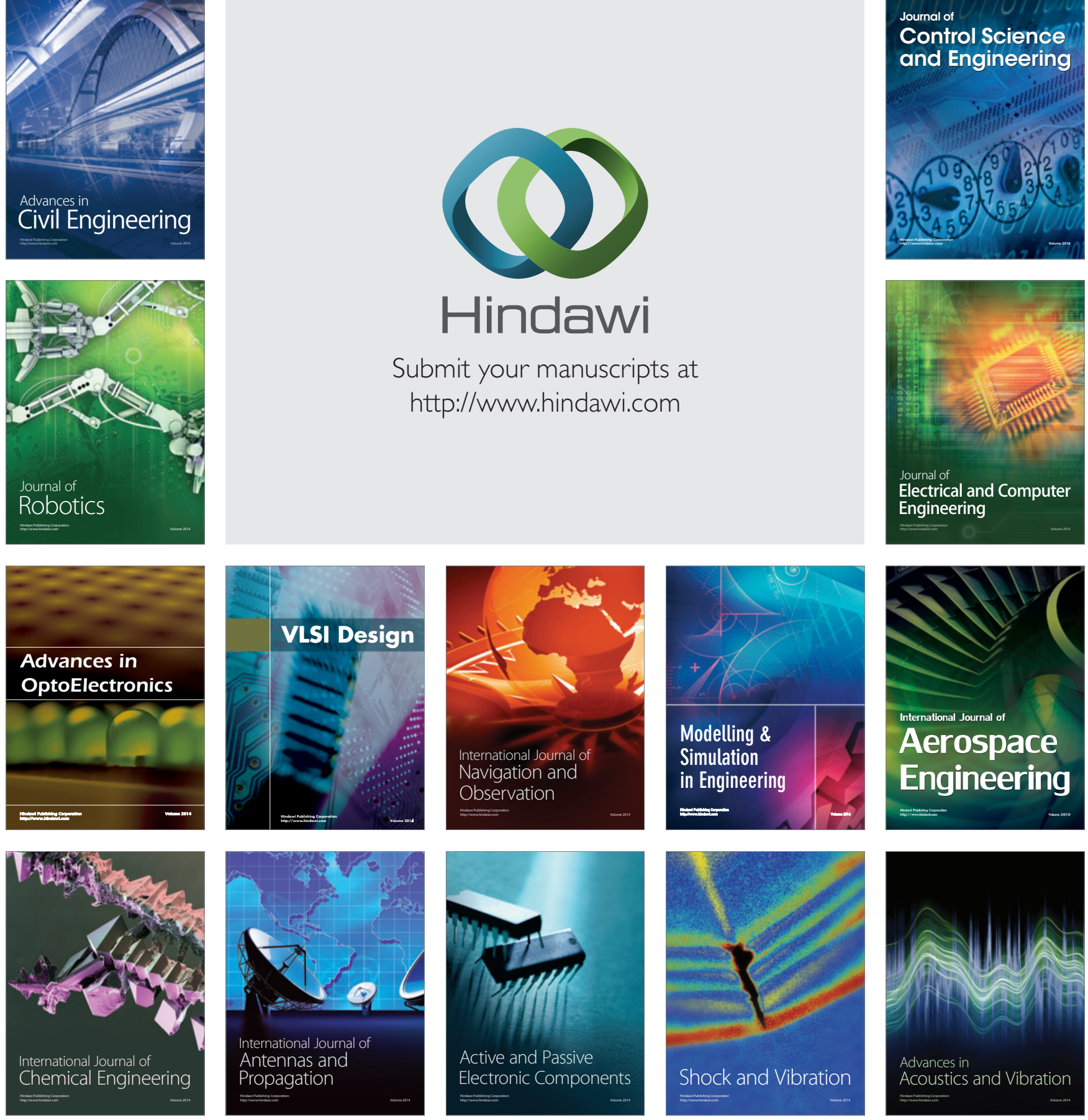